\title{
Experimental investigation of the traction force for a new space thruster
}

\author{
Yuriy Alexeevich Baurov ${ }^{1,2}$, Francesco Meneguzzo ${ }^{3,}$, Lorenzo Albanese $^{3}$ \\ ${ }^{1}$ Closed Joint Stock Company Research Institute of Cosmic Physics, 141070, Moscow Region, Pionerskaya, 4, Korolyov, Russia \\ ${ }^{2}$ Hotwater Srl , Via Gioberti, 15, I-56024 San Miniato (PI), Italy \\ ${ }^{3}$ National Research Council - Institute of Biometeorology, Via Caproni, 8, I-50145 Firenze, Italy
}

Email address:

baurov@mail.ru (Y. A. Baurov), f.meneguzzo@ibimet.cnr.it (F. Meneguzzo), 1.albanese@ibimet.cnr.it (L. Albanese)

\section{To cite this article:}

Yuriy Alexeevich Baurov, Francesco Meneguzzo, Lorenzo Albanese. Experimental Investigation of the Traction Force for a New Space Thruster. American Journal of Astronomy and Astrophysics. Special Issue: Global Anisotropy, Theory of Byuon, New Force, New Power System, Propulsion, Space Flights. Vol. 2, No. 6-1, 2014, pp.40-46. doi: 10.11648/j.ajaa.s.2014020601.16

\begin{abstract}
A long course of experimental investigations of a new non-gauge force used for the traction of a prototype space thruster placed on board an hemispherical ship are reviewed and discussed. The physical nature of the new force is explained by the theory of byuon, a non-gauge theory of the formation of the physical space and the world of ultimate particles from some unobservable objects called "byuons". The definition of these objects contains a new fundamental vector constant, the cosmological vector potential $\mathrm{A}_{\mathrm{g}}$, which direction defines a marginal global anisotropy of the physical space. The experimental results show a remarkable dependence of the force on the period of the year and even on the time of the day, the details of such dependence suggesting astronomical coordinates of the vector $\mathrm{A}_{\mathrm{g}}$ which are consistent with previous estimates yet reducing their uncertainty: in the second equatorial coordinates system, the right ascension $\alpha=316^{\circ} \pm 5^{\circ}$ and the declination $\delta=36^{\circ} \pm 10^{\circ}$ are obtained. The level of the traction force during the experimental period turns out to be in the range $2.5 \mathrm{mN}$ to $51 \mathrm{mN}$, while the best figure for the power to thrust ratio as derived from previous experiments is $330 \mathrm{~W} / \mathrm{N}$, suggestive of its potential competitiveness with state of the art electric propulsion thrusters.
\end{abstract}

Keywords: New Force, Global Anisotropy, Theory of Byuon, Traction Force, Space Thruster

\section{Introduction}

It's known to specialists working in the space industry that the realization of space vehicles to fly to distant stars using the jet principle of motion is technically impossible, at least because it would require huge amount of fuels. Even flying to the nearest planet Mars, using common technical resources of modern cosmonautics, is an extremely difficult task for humanity [1]. Therefore during the last 35 years an author of this article (YAB) developed fundamental physics in order to solve this problem. As a result, these studies paved the road to a new source of energy within the framework of the theory of byuons [2-4], i.e. a non-gauge theory of the formation of the physical space and the world of ultimate particles from unobservable objects called byuons.

The theory predicts the possibility to affect the process of formation of a fraction of the mass of ultimate particles using the potentials of physical fields, after which energy can be extracted on the basis of the usual Einstein's formula $\mathrm{E}=\mathrm{mc}^{2}$, thus opening up a practically infinite source of clean energy that can be used for the exploration of the solar system and the flight to the stars.

Since 1987 a large set of experimental researches was carried out at the best experimental bases of the USSR and Russia, and since 2012 in Italy. The first investigations were carried out at the experimental installations using a torsion balance, located in high-current magnets with fields on the order of (10-15) T [5-7]. With a body weight of about $30 \mathrm{~g}$, a force was detected at the level of $(0.7-0.8) \mathrm{mN}$. Further investigation of the new force of nature were carried out using gravimeters "Sodin" manufactured in Canada [2-4,8]; using the study of the changes in the rate of decay of radioactive elements [2-4,9-12]; plasma devices [13,14].

The existence of a new force of nature is sufficiently established and confirmed by numerous astrophysical observations such as the motion of pulsars $[15,16]$, the motion of the Sun [2,3], our star representing a natural space object carrying us under the action of the new force of nature to the constellation of Hercules, on the road cyclically reversing the polarity of its magnetic field again under the 
effect of the new force of nature, the explanation of the nature of dark energy [17], the repulsion between clusters of galaxies [17], asf.

The earlier results of the investigations on the practical implementation of the new force to generate energy and thrust for spacecrafts were show in [18]: a traction force around $0.1 \mathrm{~N}$ was detected.

Further results of the experimental investigation of the traction force carried out in November, 2012 and January to September, 2013, are shown and discussed in [19]. In [20], a project is presented to create in Mars' orbit a protective stations belt designed to reduce the asteroid hazard to the Earth, based on an interplanetary spacecraft using the new concept thruster onboard.

This article is meant to discuss investigations following the ones described in [18-20]; its specific aims are to analyze the properties of the new traction force, to discuss the methods for its further increase and to assess its value as a traction force for spacecrafts.

The paper is organized as follows: Section 2 describes the basic physical theory from which the new force of nature arises, as well as the principle of universal propulsion. In Section 3, the experimental set-up and methods for the measurement of the new traction force are shown. In Section 4 , the original results obtained during the last long run of experiments are shown and discussed, along with an original method designed to further increase the force. The main conclusions and perspectives are presented in Section 5.

\section{Usual Forces, the New Force and the Principle of Universal Propulsion}

Up to now it was assumed that only four interactions between objects in nature exist: the electromagnetic interaction, determining almost $98 \%$ the structure of all substances, the gravitational interaction, the weakest of all and determining the motion of massive bodies, the strong interaction underlying the stability of all atomic nuclei, without which they would be destroyed by the action of Coulomb forces between protons, and the weak interaction underlying the decay of many unstable elementary particles such as neutrons.

An essential distinction of the byuon theory from modern models in the classical [21] and quantum field theories [22] and the new force from other forces of nature are that the potentials of physical fields (gravitational, electromagnetic, asf.) become, in the theory of byuons, exactly fixable, measurable values using the measurements of new force.

According to the theory, a fraction of the masses of any elementary particle, up to $33 \mathrm{eV}$, is proportional to the modulus of a vectorial quantity $\mathrm{A}_{\Sigma}$ that "summarizes" the potentials of all known fields and hence will be called summary potential. $\mathrm{A}_{\Sigma}$ cannot be larger in modulus than $\mathrm{A}_{\mathrm{g}}$, a new fundamental vectorial constant appearing in the definition of non-interacting byuons: $\left|\mathrm{A}_{\mathrm{g}}\right|=1.9 \cdot 10^{5} \mathrm{~T} \cdot \mathrm{m}$. Therefore the new interaction has anisotropic properties in a wide range of scales, from that typical of the weak interaction $\left(10^{-19} \mathrm{~m}\right)$ up to the size of our Galaxy $\left(10^{22} \mathrm{~m}\right)$ and possibly even farther.

An analysis of a large run of experiments with strong magnets and torsion or piezoresonance balances carried out throughout many years (1987-19949 [1-3, 5,6], showed that the new force has a non-linear and non-local character and can be represented as a complex series (sum) in terms of changes in the summary potential $A_{\Sigma}$. The first term of such series is represented in Eq. (1):

$$
\mathrm{F}=2 \mathrm{Nm}_{\mathrm{v}} \mathrm{c}^{2} \lambda_{1}^{2} \Delta \mathrm{A}_{\Sigma}\left[\Delta\left(\Delta \mathrm{A}_{\Sigma}\right) / \Delta \mathrm{x}\right]
$$

where $\mathrm{N}$ is the number of stable particles (electrons, protons, and neutrons) in the test body, $\Delta \mathrm{A}_{\Sigma}$ is the difference in changes of the summary potential $A_{\Sigma}$ at the location points of a test body and sensor element, $\Delta\left(\Delta \mathrm{A}_{\Sigma}\right) / \Delta \mathrm{x}$ is the gradient in space of the difference potentials $\Delta \mathrm{A}_{\Sigma} ; \mathrm{x}$ is the general spatial coordinate ( $\Delta \mathrm{x}$ can be the length of an arc of a circle, or the characteristic size of the test body, according to the specific experiments); $2 \mathrm{~m}_{\mathrm{v}} \mathrm{c}^{2}=33 \mathrm{eV} ; \lambda_{1}=10^{-6}(\mathrm{Tm})^{-1}$ is the first coefficient of the series [1-3].

The detailed investigation of the new force with the aid of gravimeters one of which had an attached magnet as an amplifier of the new force [2-4,8], using the study of the changes in the rate of decay of radioactive elements [2-4,912] and of three different plasma devices at different experimental sites in different Institutes $[13,14]$ showed that the new force rejects the substance out of the region with weakened summary potential $\mathrm{A}_{\Sigma}$ along the generatrix of a cone with an opening of $100^{\circ}$ around the cosmological vector potential $\mathrm{A}_{\mathrm{g}}$ having the coordinates of right ascension $\alpha \approx 293^{\circ} \pm 10^{\circ}$ and declination $\delta \approx 36^{\circ} \pm 10^{\circ}$ in the second equatorial coordinate system. The investigation of the direction of arrival of cosmic ray (CR) particles [25,26] allowed to refine the previous estimate: $\alpha=300^{\circ} \pm 10^{\circ}$, $\delta=36^{\circ} \pm 10^{\circ}$ because the CR give the information about the vector $A_{g}$ at the scale of our Universe in the event that of ultrahigh energy CR.

The new force can be harnessed to implement a new principle of universal propulsion to move any object in any medium, based upon the role of the physical space as an environment supporting the motion of an object; the physical space is to be meant as a quantum medium filled with "hot" and "cold" dark matter $[1-3,16]$ in the framework of the theory of byuon. Therefore, it is possible to figure out some new universal class of thrusters able to move any object in any environment, e.g. under the water, on the water and through the air, as well as in the cosmic space, because it will exchange momentum neither with any ordinary substance, nor with any substance ejected from the object itself such as exhaust gases or ionized particles, rather with the physical space that in the theory of byuon is not just some mathematical fiction: it's a physical object which density of matter without ordinary substance is around $10^{-29} \mathrm{~g} / \mathrm{cm}^{3}$ ("cold" dark matter). Close to any usual material bodies, the density of matter is much larger ("warm" dark matter).

Any material body can only decrease the summary 
potential $A_{\Sigma}$ by means of the whole set of its physical potentials [1-3]: in the physical space a sort of "potential hole" arises with a reduced module of $\mathrm{A}_{\Sigma}$ that will be indicated as the information object (IO) of the given body in the physical space. The reality of the IO in the physical space was investigated for the first time by means of experiments with a physical pendulum, earlier described in [17]; in the course of 600 experiments, the new force was detected and its intensity acting upon the pendulum's load with mass $28 \mathrm{~g}$ was $1.3 \cdot 10^{-3} \mathrm{~N}$ to $4.6 \cdot 10^{-3} \mathrm{~N}$; moreover, it was established that the IO of the same body could arise in a time not longer than $0.3 \mathrm{~s}$ and vanished after not more than $7 \mathrm{~s}$.

\section{Experimental Set-Up and Method of Investigation}

The experimental set-up and the methods of investigation of the traction force generated by a simple thruster on board an hemispherical ship were described in detail in [19]. In this article we recall only its basic features.

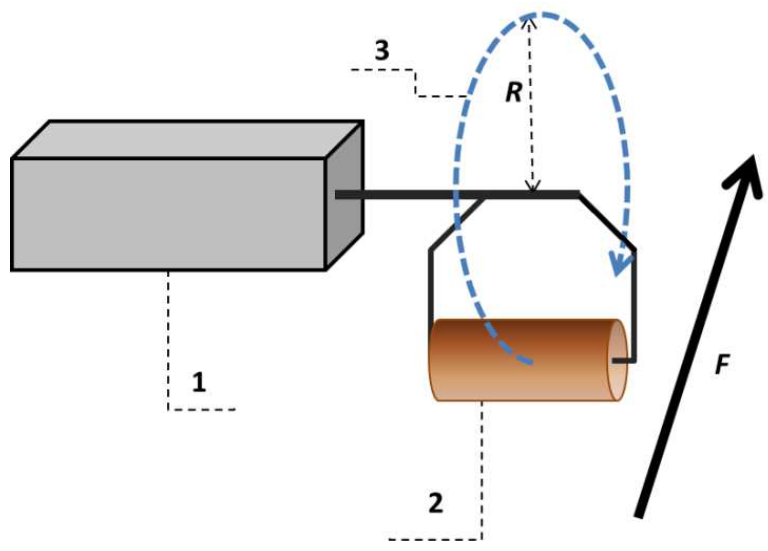

Fig. 1. Schematic view of the new thruster. 1 - electromotor (i.e. a step motor); 2 - off-center iron load; 3 - rotational trajectory of the load, periodically resting along or near the vertical; $R$ - revolution radius, not less than $5 \mathrm{~cm} ; \mathrm{F}$ - expected vector of action of the new force, producing cyclic impulses just before each stop of the load, opposite to the direction of motion of the load itself.

Fig. 1 shows the basic elements of the prototype thruster, including a programmable electro-motor, a simple mechanical device nested into the motor's shaft and in turn carrying an off-center cylinder-shape iron load performing revolutions on a vertical plane orthogonal to the motor's shaft at a distance not less than $5 \mathrm{~cm}$ from it. The motor allowed to regulate the load's revolution period, its cyclic stop in the same position, as well as the load's rest time in the stop position.

Although few experiments were performed with off-center loads having different masses (526 g, $934 \mathrm{~g}$ and $2051 \mathrm{~g}$ ) [19], in the following only the experiments performed with a load with mass equal to $526 \mathrm{~g}$, including supports and bolts, will be described. The longitudinal axis of load revolution was fixed at a distance of about $8.1 \mathrm{~cm}$ from the motor's shaft. The period of load revolution was equal to $0.22 \mathrm{~s}$ and the residence time in the stop position was $0.12 \mathrm{~s}$ in the period February 6, 2013 to June 27, 2013. Later on ad till February 28,2014 , both the period of load revolution and the residence time in the stop position were fixed to $0.3 \mathrm{~s}$.

Since the maximum action of the new force arises in few tenths of degrees $\left(10^{\circ}-30^{\circ}\right)$ before the stop position with direction tangent to the load's motion trajectory [18], the horizontal component of the impulsive force expected to be received with the load stopping in the lowest position will be significantly lower than its absolute value.

A servo-motor model DSM5.32 with nominal torque equal to $2.4 \mathrm{Nm}$ able to perform revolution cycles with periods not smaller than $0.22 \mathrm{~s}$ was used.

The ship model for investigation of traction force consists of an hemispherical body made in polycarbonate, with the inner diameter of the sphere equal to $0.9 \mathrm{~m}$, two rigidly connected steel decks to the upper of which is fixed the programmable motor, an autonomous power supply system based on a $12 \mathrm{~V}, 30$ Ah battery connected with a transformer/inverter to power the motor with $230 \mathrm{~V}$ current, supplemented with a smaller capacity $24 \mathrm{~V}$ battery to power the encoder. The total mass was about $120 \mathrm{~kg}$. The ship model was placed in a water pool having an inner diameter equal to $3.66 \mathrm{~m}$ and a depth between $0.7 \mathrm{~m}$ and $0.9 \mathrm{~m}$.

Fig. 2 shows that the traction force is measured by means of a simple set-up including a very thin cotton thread fixed to the ship model, on one side, and to a set of known weights, on the other side; the smallest weight unit has been $0.32 \mathrm{~g}$ to $1.0 \mathrm{~g}$; the actual traction force for any set of the off-center load revolution parameters is comprised between a lower value (weight rising during at least $10 \mathrm{~s}$ ) and an upper value (weight falling during at least $10 \mathrm{~s}$ ). Each measurement of the traction is repeated at least five times, until at least $80 \%$ of the measurements fall in the same interval; moreover, any measurement started $10 \mathrm{~s}$ after motor's switch-on to allow for inertia and initial conditions effects to become negligible. The resulting traction force is corrected for the observed angle between the thread and the horizontal plane (Fig. 2), as well as to account for the friction force [19].

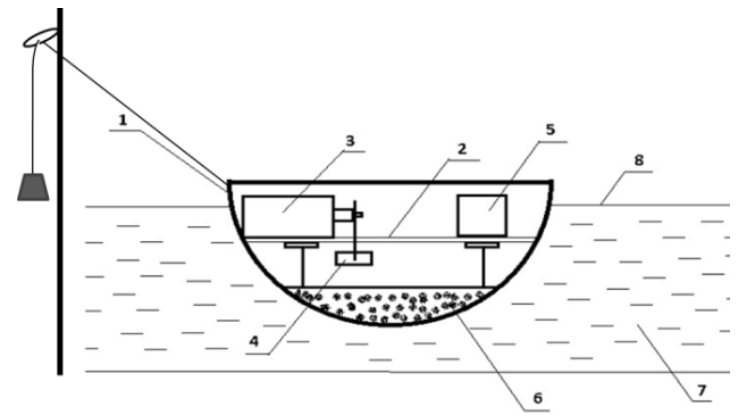

Fig. 2. Basic scheme of the model of hemispherical ship with traction generated by the new force: 1 - hemispherical housing; 2 - steel deck; 3 programmable motor; 4 - rotating load; 5 - power supply system with battery; 6 - additional load (e.g., sand); 7 -water; 8 -water surface. The traction force measurement method is also shown on the left.

The upper value of the traction force can be estimated replacing the quantities in Eq. (1) with the appropriate 
numbers; given the mass (526 g) and material (iron) of the off-center load of revolution we have for the number of stable particles: $\mathrm{N} \approx 8 \cdot 10^{26}$ and, according to $[2-4,16]$ : $\Delta \mathrm{A}_{\Sigma}=\Delta\left(\Delta \mathrm{A}_{\Sigma}\right), \Delta \mathrm{A}_{\Sigma}=\mathrm{A}_{\mathrm{g}} \cdot \cos _{\text {II I }}^{++} \cdot \mathrm{c} / \mathrm{v}, \cos _{\text {II II }}^{++}=10^{-15}$, where $\mathrm{c}$ is the speed of light, $\mathrm{v}=\Delta \mathrm{x} / \Delta \mathrm{t}=0.02 \mathrm{~m} / \mathrm{s}$ is the extinction velocity of the IO, $\Delta \mathrm{x}$ is the characteristic size of the body of revolution, i.e. around $2 \mathrm{~cm}$ in the performed experiments, and $\Delta \mathrm{t}$ is the extinction time of the IO, on the order of $1 \mathrm{~s}$ as found in [19]. Substituting into Eq. (1), the upper limit of the traction force can therefore be estimated on the order of $1 \mathrm{~N}$.

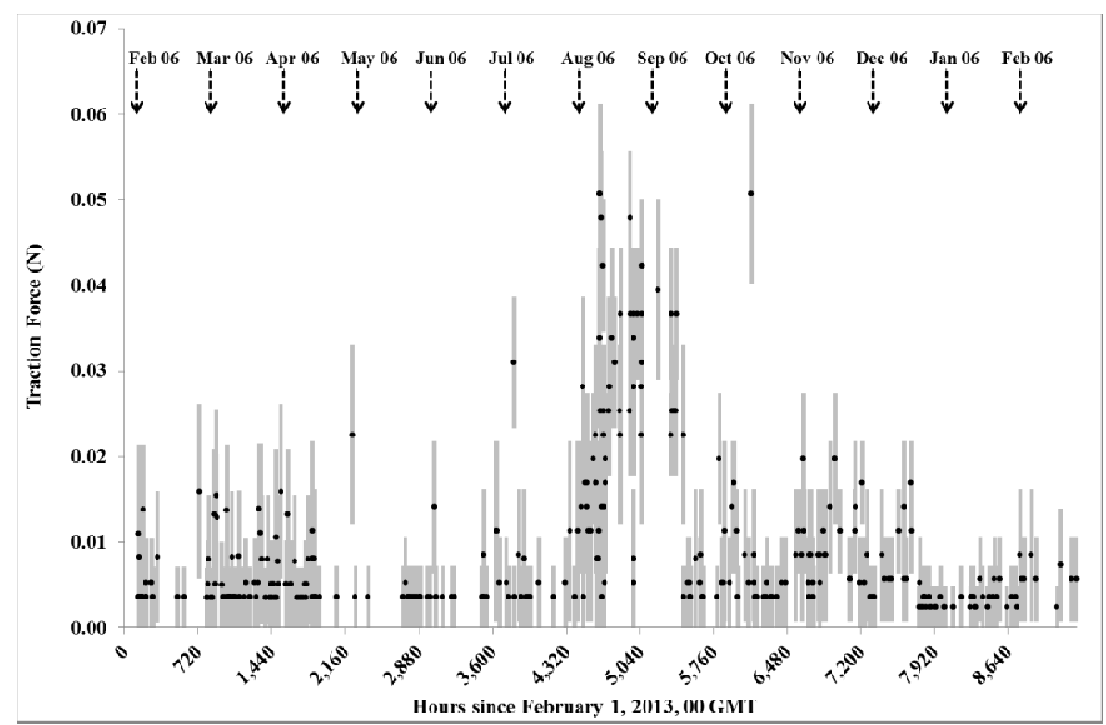

Fig. 3. Traction force data series in the period February $6^{\text {th }}, 2013-$ February 28,2014. The uncertainties associated to data points are represented as a light grey shade.

\section{Results and Discussion}

Fig. 3 shows the series of traction force data collected in the period February 6, 2013 to February 28, 2014 using the $2.4 \mathrm{Nm}$ servo-motor programmed with a revolution period and times of stop position pointed upwards. The dependence of the traction force value on time around the year is apparent Exactly on August 6, 2013, a sudden increase of the traction force is observed, such new regime lasting till October 17, 2013. The peak force in such period is around $0.05 \mathrm{~N}$. As well, the strong dependence of the traction force on the load's revolution period is shown in [19]: In the period January 27 to February 1 2013, for example, the traction force increased four times while only halving the revolution period, with as much as $0.5 \mathrm{~N}$ with a period equal to $0.12 \mathrm{~s}$.

As a consequence, while the data values were not corrected because the general applicability of the observed trend of the force with the revolution period regardless of the time of the year has not been ascertained so far, some caution is needed when comparing the numerical values of the force along the time series shown in Fig.3, having changed the revolution period from 0.22 s during February 6 to June 27, 2013, to $0.3 \mathrm{~s}$ afterwards.

In any case, the traction force appears on average considerably smaller in the period February 6 to August 6, 2013, than in the following six months.

During the earlier experimental course in January to February, 2013 [19], a sharp fall of the force, by about 5 times, occurred just around February 6, which was related to the tangential velocity of the Earth in its trajectory around the Sun. More precisely, in [19] the new hypothesis was proposed, that the IO extinction rate on the Earth is strongly dependent upon the direction of motion of our planet in the physical space around the Sun (semi-annual seasonality effect) with regards to the direction of the cosmological vector potential $A_{\mathrm{g}}$. Here, the same hypothesis is instrumental to explain the above mentioned different regimes of the traction force in the two six-months periods before and after August 6, 2013. By means of such hypothesis, the assessment of the coordinates of the vector $\mathrm{A}_{\mathrm{g}}$ in the second equatorial coordinate system can be further refined: $\alpha=316^{\circ} \pm 5^{\circ}$, $\delta=36^{\circ} \pm 10^{\circ}$.

However, in the course of experiments during the year 2014 no such sharp changes of the force around February 6, 2014 arises.

This evidence can be explained by the strong instability of the module of $A_{\Sigma}$ during 2013: it is known that the magnetic poles of the Sun finished their reconstruction around May 27, 2013, which may have contributed to the strong change of the module of $\mathrm{A}_{\Sigma}$ and, consequently, of the new force in 2013, as well as can explain the absence of the same event around February 6, 2014.

Moreover, the traction force was about zero around the vernal equinox (March 17 to 24, 2013) as well as autumnal equinox (September 18 to 22, 2013) too. Around the winter solstice (December 21, 2013) the force level was 1.2 to $1.7 \mathrm{~g}$, while around the summer solstice (June 21, 2013) annulled again.

As well, the force was practically null in the periods October 17 to 30, 2013, and December 24, 2013 to January 17, 2014 (Fig.3).

The absence of the traction force around the vernal equinox and the autumnal equinox can be explained by very 
smooth changes of module $\mathrm{A}_{\Sigma}$ by the potentials of gravitation fields from Sun and Earth. Let's note that this influence of gravitation fields on traction force in the winter solstice was steeper than in vernal solstice point and the autumnal equinox point but smoother than in summer solstice. Therefore the traction force in the winter solstice (21.12) was on level $(1.2-1.7) \mathrm{g}$ while in summer solstice (21.06) it was almost null.

The "shadow zone" (i.e., the absence) of the traction force in the period October 17 to October 30 was observed for the first time in in the experiments with a system of two quartz resonators [4]. Its physics is explained by the direction of the vector potential of magnetic field of Sun's dipole which is practically parallel with vector $A_{g}$ in this time period and consequently the changes of module $\mathrm{A}_{\Sigma}$ are minimal, as well as the new force falls to nearly zero.

The "shadow zone" from December 24 till January 17 can be explained by the influence of the solar gravitational potential because in this time our planet is flying at a minimum distance from Sun (January 4). The influence of all potentials from natural sources (Sun, Earth, etc.) creates a "stormy ocean" of $\mathrm{A}_{\Sigma}$ changes and consequently of the physical space.

On the days October 15 and 16, 2013, the traction force was significant and hourly analyses were performed, with the results shown in Fig. 4 and Fig. 5, respectively.

The lowest values of the traction force were observed at $12^{00}$ on October 15 and at $12^{45}$ on October 16 , while the peak values occurred practically at the same time on both days, i.e. around $02^{30}$. By night, the force appeared to be greater than during daylight hours. Although the statistics is clearly as yet insufficient to draw any significant conclusion, these data represent at least a clue with regards to the action of some common natural factors, i.e. regular or cyclic changes of the summary potential $A_{\Sigma}$ at the sub-daily time scale. This issue will be the subject of further research.

Space flight requires precisely known and stable values of the traction force during long time periods. As a consequence, a method was designed in order to increase the traction force especially during the "shadow zones", as well as to stabilize its value in time. The use of permanent magnets followed the basic idea that the vector potential of their magnetic fields could affect the IO extinction velocity of a load containing the magnets.

For these investigations two other identical aluminum loads were used, having diameter $\approx 10 \mathrm{~cm}$ and height $\approx 2 \mathrm{~cm}$; one of the two disks was equipped with 24 permanent magnets, each one having a diameter of $12 \mathrm{~mm}$ and a strength around 3000 Gs. The magnets were stacked at 8 equally spaced symmetric positions along the external circumference of the disk, resulting in a total mass of $530 \mathrm{~g}$. No attraction of the magnetized disk with surrounding objects was revealed. The other load was equipped with small iron disks in the same positions as the magnets in order to get the same mass.
The period of revolution was fixed at the value of $0.3 \mathrm{~s}$, the residence time in the stop position was equally $0.3 \mathrm{~s}$, while the time between consecutive experiments was always less than $15 \mathrm{~min}$ in order to minimize any other effect upon the outcomes (i.e., the traction force) different from the one possibly due to the magnetization.

The results of these experiments are shown in Fig. 6(a-b).

It's noteworthy that although the traction force was generally quite small during the study period (December 10, 2013 to February 26, 2014), the effect of magnets was generally positive and significant, except for a sub-period, namely December 24, 2013 to January 17, 2014, characterized by a particularly deep "shadow zone" (i.e. practically null force), when the growth of the force with the magnetization was very small, if any.

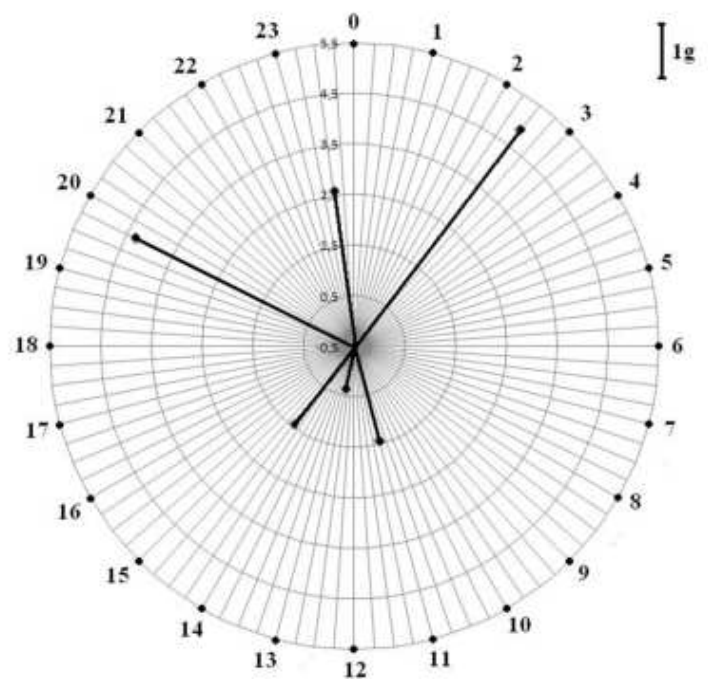

Fig. 4. Traction force on October 15, 2013. 1, 2, .., 24 - hour of day; the end points of the thick lines represent the values of the traction force.

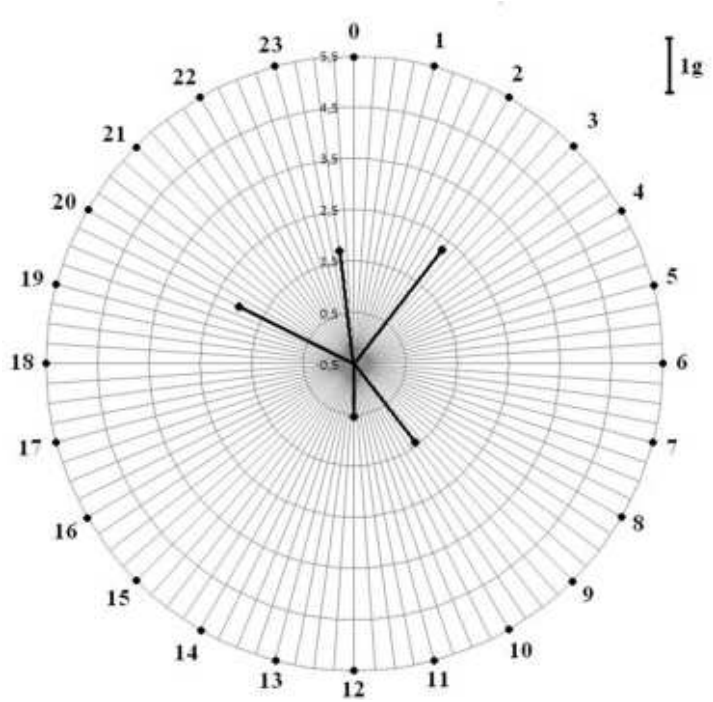

Fig. 5. Traction force on October 16, 2013. 1, 2, .., 24 - hour of day; the end points of the thick lines represent the values of the traction force. 


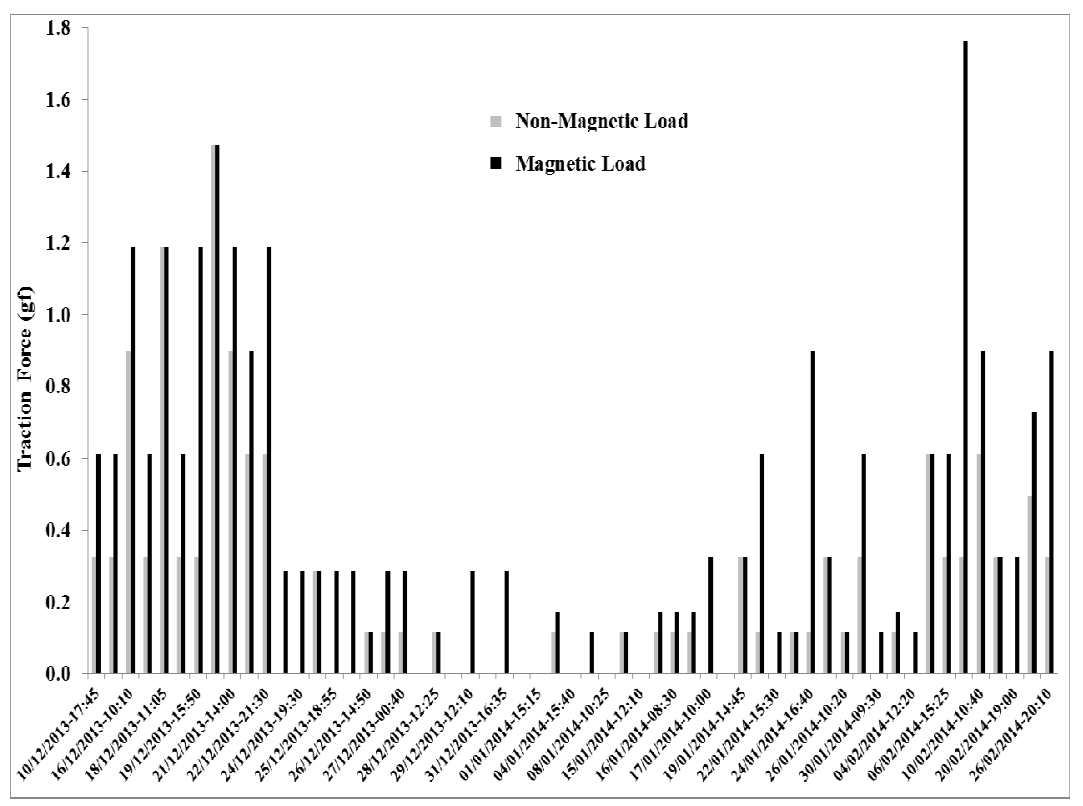

(a)

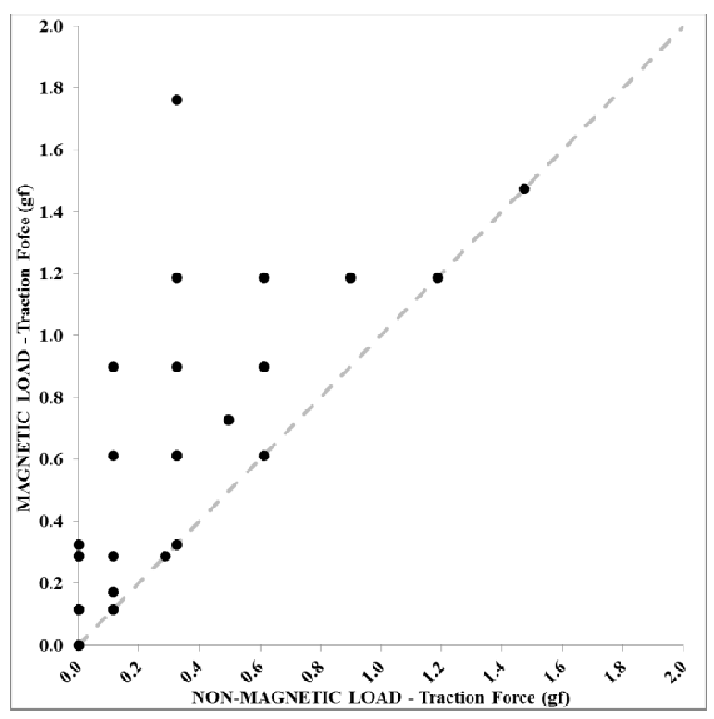

(b)

Fig. 6. The results of experimental investigations for loads with and without magnets, in units of gram-force $(102 \mathrm{~g} f=1 \mathrm{~N})$ : pairs of measurements during time (a) and scatter plot (b). Uncertainties associated to the measurements are removed and the smallest value of each measurement is shown.

\section{Conclusions}

In the opinion of the authors, the results of the described experimental runs dissipate any doubt about the existence in nature of a previously unknown interaction that, in contrast to the well-known ones, is not gauge invariant: potentials of physical fields are instrumental to its implementation, such potentials being considered as non-observable quantities in the standard physics.

The results of a long experimental run (February 6, 2013 to February 28, 2014) of new thruster traction force confirmed once again the existence of a new force of nature and the possibility to harness it for space flights [18-20].
The experiments showed the dependence of the level of the traction force on the time of the year and even on the hour of the day. The peak levels of the traction force were observed on average in the period August 6 to October 16, 2013. It is show that using magnetized loads in the thruster allows to increase and to some extent to stabilize the traction force.

The new concept thruster is a unique space-tug not requiring any fuel as well as having a very small specific power around $330 \mathrm{~W} / \mathrm{N}$ [19], i.e. about 40 times smaller than the best corresponding figures of electric propulsion thrusters used to move satellites and spacecrafts [27]; moreover, its construction is a very simple.

The new thruster can be used for any space flights, 
including the orbit raising for the International Space Station, as well as thruster onboard the interplanetary spacecraft designed to prevent the asteroid hazard to the Earth [20].

\section{Acknowledgments}

The authors gratefully acknowledge A. Yu. Baurov for help in the preparation of the manuscript; G.M. Lanini and G. Tagliaferri for assistance with the experimental stands and measurements; C. Dionisio for valuable discussions about space propulsion; M. Pagliaro for the outstanding support and motivation by means of the "SuNEC 2013" Conference.

\section{Appendix}

Abbreviations used in this article:

$\mathrm{A}_{\Sigma}=$ Summary vector potential $(\mathrm{T} \cdot \mathrm{m})$

$A_{g}=$ Cosmological vector potential $(\mathrm{T} \cdot \mathrm{m})$

$\mathrm{CR}=$ Cosmic Rays

$\mathrm{IO}=$ Information Object in the physical space

\section{References}

[1] A.S. Koroteyev, ed., Manned Mission to Mars, Russian Academy of Cosmonautics named after K.E. Tsiolkovsky, Moscow-Korolev, 2006.

[2] Yu.A. Baurov, Structure of Physical Space and New Method of Obtaining Energy (Theory, Experiment, Applications), Moscow, "Krechet" (in Russian), (1998).

[3] Yu.A. Baurov, On the structure of physical vacuum and a new interaction in Nature (Theory, Experiment and Applications), Nova Science, NY, 2000.

[4] Yu.A. Baurov, Global Anisotropy of Physical Space, Experimental and Theoretical Basis, Nova Science, NY, 2004.

[5] Yu.A. Baurov, Yu.N. Babaev, V.K. Ablekov, On one model of weak, strong and electromagnetic interactions, Dokl. Akad. Nauk. 259 (1981) 1080-1084.

[6] Yu.A. Baurov, E.Yu. Klimenko, S.I. Novikov, Experimental observations of magnetic anisotropy, Dokl. Akad. Nauk SSSR. 315 (1990) 1116-1120.

[7] Yu.A. Baurov, P.M. Ryabov, Experimental investigations of magnetic anisotropy of space using quartz piezoresonance balance, Dokl. Akad. Nauk SSSR. 326 (1992) 73-77.

[8] Yu.A. Baurov, A.V. Kopaev, Experimental investigations of signals of a new nature with the aid of two high precision stationary quartz gravimeters, Hadron. J. 25 (2002) 697-712.

[9] Yu.A. Baurov, A.A. Konradov, V.F. Kushniruk, E.A. Kuznetsov, Yu.G. Sobolev, Yu.V. Ryabov, et al., Experimental investigations of changes in beta-decay rate of $60 \mathrm{Co}$ and 137Cs, Mod. Phys. Lett. A. 16 (2001) 2089-2101.

[10] Yu.A. Baurov, Yu.G. Sobolev, Yu. V. Ryabov, V.F. Kushniruk, Experimental investigations of changes in the rate of beta decay of radioactive elements, Phys. At. Nucl. 70 (2007) $1825-1835$.
[11] Yu.A. Baurov, The anisotropic phenomenon in the $\beta$ decay of radioactive elements and in other processes in nature, Bull. Russ. Acad. Sci. Phys. 76 (2012) 1076-1080.

[12] Yu.A. Baurov, V.L. Shutov, On the influence of vectorial magnetic potential of the Earth and the Sun on the $\beta$ - decay rate, Prikl. Fiz. 1 (1995) 40-45.

[13] Yu.A. Baurov, I.B. Timofeev, V.A. Chernikov, S.F. Chalkin, A.A. Konradov, Experimental investigations of the distribution of pulsed-plasma-generator radiation at its various spatial orientation and global anisotropy of space, Phys. Lett. A. 311 (2003) 512-523.

[14] Yu.A. Baurov, A.G. Znak, V.G. Farafonov, Experimental Investigation of Heat Content in the Jet of Magnetoplasmadynamic Accelerator in Accordance with its Spatial Orientation, in: Adv. Plasma Phys. Res. v. 5, Nova Science Publishers Inc, New York, 2007: pp. 179-196.

[15] Yu.A. Baurov, A.A. Shpitalnaya, I.F. Malov, Global anisotropy of physical space and velocities of pulsars, Intl. J. Pure Appl. Phys. 1 (2005) 71-82.

[16] I.F. Malov, Yu.A. Baurov, The distribution of space velocities of radio pulsars, Astron. Reports. 51 (2007) 830-835.

[17] Yu.A. Baurov, I.F. Malov, On the Nature of Dark Matter and Dark Energy, J. Mod. Phys. 01 (2010) 17-32.

[18] Yu.A. Baurov, A.Yu. Baurov, A.Yu.J. Baurov, F. Meneguzzo, A.A. Bugaev, New Interaction in Nature and its Use in the Form of Traction, Int. J. Pure Appl. Sci. Technol. 13 (2012) $40-49$.

[19] Yu.A. Baurov, L. Albanese, F. Meneguzzo, V.A. Menshikov, Universal propulsion harnessing the global anisotropy of the physical space, Am. J. Mod. Phys. 2 (2013) 383-391.

[20] Yu.A. Baurov, L. Albanese, F. Meneguzzo, V.A. Menshikov, Protecting the Planet from the Asteroid Hazard, Int. J. Pure Appl. Phys. 9 (2013) 151-168.

[21] L.D. Landau, E.M. Lifshitz, The Classical Theory of Fields, Volume 2, 1962.

[22] N.N. Bogolyubov, D.V. Shirkov, Introduction to the theory of quantized fields, Nauka, Moscow, 1984.

[23] Yu.A. Baurov, E.Yu. Klimenko, S.I. Novikov, Experimental observation of space magnetic anisotropy, Phys. Lett. A. 162 (1992) 32-34.

[24] Yu.A. Baurov, Space magnetic anisotropy and a new interaction in nature, Phys. Lett. A. 181 (1993) 283-288.

[25] Yu.A. Baurov, The Anisotropy of Cosmic Rays and the Global Anisotropy of Physical Space, J. Mod. Phys. 03 (2012) 17441748 .

[26] Yu.A. Baurov, Research of global anisotropy of physical space based on investigation of changes in $\beta$ and $\alpha$-decay rate of radioactive elements, motion of pulsars and anisotropy of cosmic rays, Am. J. Mod. Phys. 2 (2013) 177-184.

[27] M. Dudeck, F. Doveil, N. Arcis, S. Zurbach, Plasma propulsion for geostationary satellites for telecommunication and interplanetary missions, IOP Conf. Ser. Mater. Sci. Eng. 29 (2012) 012010. 